Univ.Prof. Dr. Peter Hackl

\title{
Wünsche an ein Österreichisches Bundesstatistik-Gesetz
}

\author{
Ergebnisse einer ÖSG-Podiumsdiskussion \\ am 7. Oktober 1997
}

\section{Einleitung}

Die Österreichische Statistische Gesellschaft, Vereinigung von etwa 600 Berufsstatistikern, hat im Rahmen ihrer außerordentlichen Hauptversammlung ihre Erwartungen betreffend ein neues Bundesstatistik-Gesetz diskutiert. Die Impulsreferate wurden (in alphabetischer Folge) von

- Univ.Prof. Dr. Peter Bauer (Institut für Medizinische Statistik und Dokumentation, Universität Wien) als Vertreter der universitären Statistik,

- w.Hofrat Dr. Ewald Kutzenberger (Statistischer Dienst, Amt der Oberösterreichischen Landesregierung) als Vertreter der amtlichen Statistik, und

- Dr. Josef Richter (Statistische Abteilung, Wirtschaftskammer Österreich) als Vertreter der angewandten Statistik

gehalten und sind in den folgenden drei Abschnitten wiedergegeben. Die in den Impulsreferaten geäußerten Wünsche und Argumente wurden von den Diskussionsteilnehmern aus dem Auditorium großteils positiv kommentiert und unterstützt. Die Sorge, daß die Zielsetzung und Tätigkeit der amtlichen Statistik den Möglichkeiten und Notwendigkeiten der Zeit und Zukunft angepaßt werden, wurde in fast allen Wortmeldungen deutlich. Sie muß als Ausdruck der Verantwortung interpretiert werden, die beruflich mit Statistik befaßte Experten für die Qualität der von der amtlichen Statistik produzierten Statistiken und der darauf basierenden Entscheidungen in Politik, Wirtschaft und Verwaltung empfinden.

Als Forderungen formuliert, erwarten die Berufsstatistiker Österreichs, daß folgende Punkte beachtet werden:

Das Bundesstatistik-Gesetz soll die Tätigkeit der Amtlichen Statistik als eine Infrastrukturleistung für Politik, Wirtschaft, Verwaltung und Öffentlichkeit mit wissenschaftlichem Charakter anerkennen. 
Die Schaffung eines neuen Bundesstatistik-Gesetzes eröffnet die Möglichkeit, die Rolle der amtlichen Statistik für das Funktionieren eines modernen Staates und die hohen Anforderungen an ihre Arbeitsweise festzuschreiben. Amtliche Statistik ist in einem modernen Staat nicht Mittel der Verwaltung sondern Basis für Entscheidungen in allen Bereichen des öffentlichen und privaten Lebens. Der wissenschaftliche Charakter der Tätigkeit des Statistikers als Voraussetzung der Qualitätsicherung der statistischen Ergebnisse wird etwa auch in der Verordnung der EC über Gemeinschaftsstatistiken oder im Arzneimittelgesetz anerkannt. Die Berücksichtigung dieses Anspruches für die amtliche Statistik kann nicht hoch genug eingeschätzt werden und wird die Vertreter der amtlichen Statistik in die Lage versetzen, ihren Forderungen nach entsprechenden Ressourcen neues Gewicht zu geben. Die Berücksichtigung dieses Anspruches schließt aber auch ein, daß unabhängige Experten für statistische Methoden in die Gestaltung des neuen Bundesstatistik-Gesetzes einbezogen werden, und diese nicht Juristen und Repräsentanten des bestehenden Systems alleine überantwortet wird.

Das Bundesstatistik-Gesetz soll als allgemeine Zielsetzung die Bestrebungen festhalten, (a) zukunftsorientiert und innovativ die Qualität und Aktualität von Ergebnissen der Amtlichen Statistik zu erhöhen und (b) Möglichkeiten eröffnen, die Belastung der Auskunftgeber zu verringern.

Diese Forderung schließt eng an die erste an und verlangt Zielsetzungen für die Qualität der statistischen Ergebnisse und für die Interaktion mit den Auskunftgebern. Vier Detailforderungen, die im folgenden genannt werden, konkretisieren diese allgemeinen Zielsetzungen und zeigen Möglichkeiten ihrer Umsetzung. Die wesentlichen Forderungen betreffen die Offenlegung der verwendeten Daten und Methoden und die Erleichterung des Zuganges zu Verwaltungsdaten zwecks Entlastung der Respondenten.

Schließlich ist explizit die Forderung nach dem Einbeziehen von unabhängigen Experten für statistische Methoden in die Gestaltung des Bundesstatistik-Gesetzes zu stellen. Es ist eine Selbstverständlichkeit, daß beispielsweise in die Beratungen eines Gesetzes mit medizinischer Relevanz von der Ärztekammer nominierte Experten zugezogen werden. Ebenso selbstverständlich sollte das im vorliegenden Fall gelten. Die administrative Erschwernis, einen oder mehrere Gesprächspartner berücksichtigen zu müssen, wird sicherlich durch die Chance aufgewogen, durch den Beitrag von Statistik-Experten zu einem besseren Gesetz zu kommen.

Die Österreichische Statistische Gesellschaft verlangt, daß in die Gestaltung des Bundesstatistik-Gesetzes unabhängige Experten für statistische Methoden einbezogen werden, und ist bereit, entsprechende Experten zu nominieren. 


\section{Die Sicht des universitären Statistikers}

von Peter Bauer ${ }^{1}$

Ziel dieses Beitrags ist es, für das eng umschriebene Gebiet der Entwicklung und Zulassung neuer Arzneimittel oder Medizinprodukte aufzuzeigen, in welch vielfältiger Weise die Statistik als wissenschaftliche Disziplin in Gesetze, Guidelines usw. in Österreich, aber auch international integriert ist. In diesem Gebiet fokussieren viele verschiedene Interessen, sodaß dort der Bedarf nach Regelungen besonders groß ist. Dies könnte Anregungen für die Formulierung des Statistikgesetzes liefern.

Die allgemeinste Norm, aus der der Biostatistiker die Notwendigkeit der Anwendung statistischer Verfahren auf dem aktuellen wissenschaftlichen Stand ableitet, ist Punkt 1 in den allgemeinen Grundsätzen der Deklaration von Helsinki (Anhang 1). Es ist wichtig zu erwähnen, daß auch in den Grundsätzen im Kapitel III Artikel 10 der Verordnung des Rates über die Gemeinschaftsstatistiken für die EU "das Heranziehen wissenschaftlicher Kriterien" verlangt wird (Anhang 1).

Im österreichischen Arzneimittelgesetz wird im $\S 35$ gefordert, daß der "Prüfer" Kenntnisse auf dem einschlägigen Gebiet der Biometrie verfügen, oder einschlägige Fachkräfte mitverantwortlich beiziehen soll (Anhang 2). Im $\S 36$ wird explizit Transparenz der erhobenen Daten für Außenstehende verlangt (Anhang 3). Im $\S 37$ ist die wichtige Rolle der Biometrie bei der Festlegung des Prüfplans dokumentiert. Dort steht auch, daß die Art der statistischen Analyse, die angewendet werden soll, schon vor Beginn des Experiments im Prüfplan ausgeführt sein muß. Dies ist ein wichtiger Beitrag zur Methodentransparenz (Anhang 4). Schließlich werden klare Verantwortlichkeiten eines qualifizierten Biometrikers für die Planung und Ausführung der statistischen Analyse festgeschrieben (Anhang $5)$.

Um den Stand der Wissenschaft auf dem Gebiet der Biometrie bei klinischen Versuchen einzugrenzen, gibt es zusätzlich internationale Richtlinien mit zum Teil sehr hohem Detaillierungsgrad. Die für die Biometrie bedeutendste Richtlinie ist die aus den einzelnen Regelungen in Europa, USA und Japan international entstandene ICH-Guideline Statistical Principles for Clinical Trials. Die Zahl der Guidelines in diesem Bereich wird ständig größer, wobei auch sehr spezielle Fragen behandelt werden (Anhang 6). Bei der Entstehung dieser Guidelines waren die betroffenen Statistiker der Ämter, der Industrie und der Universitäten beteiligt. Es gab Stellungnahmen zu den Entwürfen von verschiedensten statistischen Fachgesellschaften und Interessensgruppen. Die der Formulierung der ICH-Guidelines vornehmlich zugrundeliegende europäische Regelung wurde seinerzeit auf Initiative Einzelner von Anfang an in Kooperation mit Personen aus den verschiedenen Bereichen erstellt. Das Inhaltsverzeichnis der ICH-Guideline zeigt, wie vielfältig die Bereiche sind, zu denen Stellung genommen wird (Anhang 7). Als Beispiel für die Art der Formulierung sei Kapitel 4.4 zu der sehr speziellen Frage der Änderung des ursprünglich vorgesehenen Stichprobenumfangs angeführt (Anhang 8).

Aus meiner Erfahrung bei der Mitwirkung für die einschlägige Europäische "Note for

${ }^{1}$ Univ.Prof. Dr. Peter Bauer, Institut für Medizinische Statistik und Dokumentation, Universität Wien 
Guidance” seien folgende Wünsche an das Statistikgesetz formuliert:

1. Da es sich bei dem neuen Österreichischen Statistikgesetz um die Weichenstellung für viele Jahre in einem für den Staat und seine Bürger immer wichtiger werdenden Bereich handelt, ist dieses Gesetz von vornherein in Kooperation aller Betroffenen $\mathrm{zu}$ formulieren.

2. Der grundsätzlich wissenschaftliche Charakter statistischer Methoden und die Verpflichtung, dem jeweiligen Stand der Wissenschaften gemäße Verfahren anzuwenden, sind im Gesetz zu berücksichtigen.

3. Die ÖSG als Fachgesellschaft der österreichischen Statistiker ist so früh als möglich in den Entstehungsprozeß des Gesetzes einzubinden. Die Forderung, bei der Neuschaffung des Statistikgesetzes möglichst frühzeitig Personen miteinzuschalten, die nicht nur der betroffenen Beamtenschaft angehören, ist getragen von der Sorge, daß das neue Statistikgesetz ohne Visionen für die Positionierung der Statistik in der kommenden Informationsgesellschaft nicht die dringend notwendigen Impulse setzen kann.

\section{Die Sicht des amtlichen Statistikers}

\section{von Ewald Kutzenberger ${ }^{2}$}

Es ist mir ein Anliegen Ihnen zu sagen, daß ich hier nicht als akkordierter Sprecher der Landesstatistiker oder gar der Amtlichen Statistik in Österreich auftrete. Ich bringe hier lediglich meine langjährige Erfahrung als Landesstatistiker von Oberösterreich und aus einem intensiven Befassen mit der Amtlichen Statistik als Lektor für dieses Fach an der Universität Linz ein.

Nun zu meinen Wünschen:

1. Das Bundesstatistikgesetz stammt noch aus einer Zeit, in der die Verwaltung noch keine EDV verwendet hat. Die einzigen, die damals schon modern waren, sind die Amtlichen Statistiker, die schon seit 1891 einen programmierbaren Zählautomaten mit Lochkarten verwendeten. Damals eine Weltsensation. Oder 1973: Die Datenbank ISIS, eine international beachtenswerte Entwicklung. Und seither - nichts mehr. Es tut mir irgendwie leid, mitansehen zu müssen, daß die Amtliche Statistik in Österreich gerade in den letzten Jahren diese Vorreiterrolle, die sie einmal hatte, total verspielt hat.

Aber zurück zum Statistikgesetz. Nachdem es zur Zeit der Formulierung des gültigen Gesetzes keine EDV-geführten Verwaltungsinformationen gab, hat das Gesetz die Möglichkeit der statistischen Verarbeitung dieser Informationen auch nicht vorgesehen. Das führt dazu, daß eine ungeheure Fülle von Informationen für statistische Zwecke nicht zugänglich ist und, statistisch gesehen, brach liegt. Eine unvorstellbare Vergeudung von Wissen. Stellen Sie sich einen Konzern vor, der die

\footnotetext{
${ }^{2}$ w.Hofrat Dr. Ewald Kutzenberger, Statistischer Dienst, Amt der Oberösterreichischen Landesregierung
} 
Informationen aus seinen Teilbetrieben einfach liegen läßt und sie nicht für eine Führungsinformation verwendet. - Unvorstellbar.

Was will eigentlich die Amtliche Statistik: Sie soll

- die gesellschaftliche, wirtschaftliche und ökologische Entwicklung beobachten,

- auftretende Probleme möglichst früh erkennen und

- Grundlagen für die Planung, für Entscheidungen und für die Kontrolle von Maßnahmen liefern.

Und das alles ohne gesetzlich abgesicherten Zugang zu Verwaltungsdaten. Eine logische Schlußfolgerung in einem modernen Staat mit einem verantwortungsvollem Controlling kann nur sein:

In jedem Gesetz, durch dessen Vollziehung die Verwaltung Informationen erhält, ist eine Regelung aufzunehmen, daß diese Informationen zur anonymen statistischen Verarbeitung an die Amtliche Statistik weiterzuleiten sind. Verknüpfungen dieser Daten in der Kapsel der streng abzuschottenden Amtlichen Statistik sind erlaubt.

Im Bundesstatistikgesetz ist zu regeln, daß all diese Daten aus der Verwaltung streng anonymisiert ausschließlich für statistische Zwecke verarbeitet, also auch verknüpft werden dürfen. Dies führt zu einem Quantensprung in der Qualität der Amtlichen Statistik bei ungleich geringerer Belastung der Bevölkerung und bei ungleich geringeren Kosten. Denken sie nur, daß zum Beispiel die überaus unangenehmen Befragungen zum Einkommen total wegfallen würden und sich die Qualität der Einkommensstatistiken um ein Vielfaches verbessern würde.

2. Um den Bürgern Sicherheit gegen Mißbrauch dieser übermittelten Daten zu geben, sind Maßnahmen erforderlich. Zum ersten muß gesagt werden, daß etwa bei einer Befragung (denken Sie an Einkommensfragen) ungleich mehr Personen mit den - noch gar nicht anonymisierten Informationen - in Berührung kommen, als etwa bei einer automatisierten und anonymisierten Übermittlung an die Amtliche Statistik durch das Finanzministerium. Eine Unsicherheit im Schutz der Daten der Amtlichen Statistik muß bei dieser Gelegenheit jedoch unbedingt beseitigt werden. Die Übermittlung aller an die Amtliche Statistik übermittelten Daten kann - ich zitiere hier Dr. Richter - nur eine Einbahnstraße in Richtung Statistik sein. Es darf keine, ich betone, keine wie immer geartete Möglichkeit geben, Individualdaten aus der Kapsel Amtliche Statistik herauszubekommen.

Nach der derzeitigen gesetzlichen Regelung gibt es aber diesen Weg: Legt die Erhebungsverordnung fest, daß die Daten nicht nur zu statistischen Zwecken verwendet werden dürfen, sondern auch für andere gesetzlich geregelte Zwecke der Verwaltung, so dürfen die Daten weitergegeben werden. Wenn Sie das Beispiel des LFBIS nehmen, in dem der Minister einen Freibrief hat, mit den personenbezogenen (!!!) 
Daten zu tun, was er will, dann kann das kein ausreichender Schutz vor mißbräuchlicher Verwendung der Daten sein. Diese Möglichkeit muß ein für alle mal ausgeschaltet werden, um eine Basis für eine umfassende und sichere Verarbeitung von Verwaltungsdaten zu schaffen.

3. $\mathrm{Zu}$ diskutieren ist hier auch der Politikereinfluß auf die Amtliche Statistik: Ist die Amtliche Statistik sozusagen der Sammeltopf, in dem alle anonymisierten Daten der Verwaltung zur statistischen Beobachtung zusammenlaufen, so muß unbedingt die Möglichkeit ausgeschaltet werden, daß etwa ein für das Ressort zuständiger Politiker Zugang zu diesen Einzeldaten hat oder gar Weisungen für die Verarbeitung erteilen kann.

In der EU werden Modelle für die Integration der Amtlichen Statistik in einem Staatsverband erarbeitet, in denen dieser ungewollte politische Einfluß ausgeschlossen ist. Vorbild kann etwa der Rechnungshof sein. Auch hier sollte ein neues modernes Bundesstatistikgesetz klare Regelungen anbieten.

4. Der letzte Wunsch, den ich hier artikulieren möchte, betrifft den Anhang des Bundesstatistikgesetzes. In ihm sind alle Erhebungsgegenstände angeführt, für die eine statistische Erhebung mit Verordnung angeordnet werden kann. Für jeden dieser Gegenstände sind auch alle Merkmale die erhoben werden dürfen angeführt. Das heißt: ist die Erhebung eines hier nicht angeführten Merkmales notwendig, muß dazu ein eigenes Gesetz beschlossen werden. Ich denke, daß wir in unserer schnellebigen Informationsgesellschaft heute gar nicht vorausdenken können, welche Merkmale etwa in fünf oder in zehn Jahren zur Beobachtung unserer Gesellschaft notwendig sein werden. Wir sollten diese Beschränkung auf vorgedachte Merkmale im Bundesstatistikgesetz fallen lassen. Es passiert dadurch gar nichts. Es muß ohnehin für die konkrete Erhebung eine Durchführungsverordnung erlassen werden, in der die Merkmale angeführt werden. Das Gesetz erreicht nur eine ungleich höhere Flexibilität und es bleibt länger anwendbar, ohne daß dadurch Mißbrauch getrieben werden könnte. Wir haben in Oberösterreich diese Regelung im Landesstatistikgesetz nach anfänglichem Widerstand des hohen Datenschutzrates doch erreicht und die beste Erfahrung damit gemacht.

Sehr geehrte Damen und Herren, das sind die Wünsche eines kleinen Landesstatistikers an die große Bundesstatistik. Es würde mich sehr freuen, wenn sie nicht ganz ungehört blieben. 


\section{Die Sicht des angewandten Statistikers}

von Josef Richter ${ }^{3}$

Aus der Sicht des angewandten Statistikers seien folgende Wünsche an ein neues Bundesstatistik-Gesetz formuliert:

1. Weitere und explizite Fassung der Ziele und der Aufgaben der amtlichen Statistik ("Paradigmenwechsel").

Die Aufgabe der amtlichen Statistik kann es nicht nur sein, wie die Europäische VO im Artikel 1 festlegt, "Grundlagen für die Durchführung, Beobachtung und Bewertung der Gemeinschaftspolitik" zu liefern. Auf nationale Ebene übertragen würde das heißen, "statistische Daten für die öffentliche Verwaltung bereitstellen", und irgendwo - wenn auch nicht explizit - ist diese Sicht der Dinge im derzeitigen Bundesstatistikgesetz auch verankert. Die amtliche Statistik hat aber inzwischen in der Informationsgesellschaft eine ganz andere Rolle übernommen, und das muß auch im Gesetz seinen Niederschlag finden. Vor der derzeitigen engen Sicht der Funktion der amtlichen Statistik - Hilfsfunktion für die Verwaltung - läßt sich weder der Aufwand vertreten, der dem öffentlichen Sektor im Bereich der amtlichen Statistik erwächst, noch der viel größere Aufwand, der unzähligen Bürgern und ebenso unzähligen Betrieben zugemutet wird. Solche Belastungen sind nur zu vertreten, wenn Statistik als öffentliches Gut im allgemeinen Interesse gesehen wird. Diese geänderte Sicht ist ein Paradigmenwechsel, der hier eingefordert werden soll.

Ebenso wichtig ist es, den wissenschaftlichen Charakter der Statistik klarzustellen. Dieser Charakter sollte deutlicher und prominenter herausgestellt werden, als es die EU-Verordnung unter dem Titel "Zuverlässlichkeit" tut. Dort heißt es in Artikel 10, daß die Statistiken die Gegebenheiten möglichst genau widerspiegeln sollen, wobei wissenschaftliche Kriterien bei der Wahl der Quellen Methoden und Verfahren heranzuziehen sind.

Ich meine, daß der Aspekt, daß es sich bei Statistik grundsätzlich um eine wissenschaftliche Aufgabe handelt, nämlich um den Versuch, Wirklichkeit im Blick auf ein analytisches Ziel möglichst adäquat zu erfassen, d.h. um einen bewußten Akt, den Versuch der Apperzeption, in einem Statistikgesetz explizit präsent sein müßte. Eine solche Deklaration würde auch helfen, falsche Erwartungshaltungen (z.B. nach Punktlösungen) bei den Benutzern und Auftraggebern auszuschalten.

\section{Klare und umfassende Festlegung der Publikationspflicht}

Eng verbunden mit dem 1. Wunsch und der Sicht der amtlichen Statistik als ein öffentliches Gut heißt dies auch, daß die Publikationspflicht klar und deutlich gefaßt wird.

Das derzeitige Statistikgesetz widmet der Publikationspflicht nur einen Absatz eines Paragraphen und meint nur "Die Ergebnisse der Erhebungen sind in solcher

${ }^{3}$ Dr. Josef Richter, Statistische Abteilung, Wirtschaftskammer Österreich 
Weise zu veröffentlichen...” Der Akzent liegt also mehr auf der Art der Veröffentlichung als auf der grundlegenden Pflicht zu veröffentlichen selbst. Drei Facetten des Zugangs zu den Ergebnissen erscheinen mir unverzichtbar:

- der österreichische Konsument im allgemeinen Sinn darf nicht schlechter gestellt werden als die Behörde, sei es die österreichische Bundesregierung oder die europäische Kommission

- was die Medien betrifft, muß ebenfalls ein fairer Zugang möglich sein; Verfügbarmachen in elektronischen Medien, die im Inland nur 100 Benutzern zugänglich sind, erfüllt nicht die Publikationspflicht

- was die Kosten betrifft: die Publikationspolitik und Preispolitik von EUROSTAT soll hier nicht als Vorbild, sondern als abschreckendes Beispiel dienen.

Auch die Sicht der Statistik als öffentliches Gut gibt hier ein Argument: Wenn Bürger und Firmen zur Mitwirkung ohne Kostenersatz verpflichtet werden, dann steht ihnen auch das Recht des Zuganges zu Daten zu.

In der Publikationspolitik (z.B. Zeitpunkt der Publikation, Umfang) ist der politische Einfluß ebenso auszuschalten wie bei der Wahl der Methodik.

\section{Pflicht zur "Produktdeklaration"}

Die europäische Statistik VO fordert Transparenz nur bezüglich

- der Rechtsgrundlagen,

- der Zwecke und

- der vorgesehenen Datenschutzmaßnahmen.

Was fehlt, ist die Pflicht zur Bereitstellung aller jener Informationen methodischer, technischer Art, ohne die ein statistisches Ergebnis nicht sinnvoll interpretiert werden kann. Den Erstellern amtlicher Statistik ist die Bereitstellung dieser Information im Gesetz zur Pflicht zu machen. Sie wäre bei allen Produkten so zwingend vorzusehen wie der Beipackzettel bei Medikamenten, der

- für den Experten, den Arzt, die Zusammensetzung des Medikaments angibt und

- für den Laien verständlich Indikation und Kontraindikation anführt.

In den Vereinigten Staaten etwa besteht der Freedom of Information Act, der jedem Bürger das Recht einräumt, über jede staatliche Tätigkeit Auskunft einzuholen. Wie ich mich vergewissern konnte, wird diese Bestimmung von den Konsumenten der Statistik genutzt, um von den vielen Stellen, die in den Vereinigten Staaten amtliche Statistik betreiben, detaillierte Auskünfte über alle technischen Details, die gewählten Methoden, Stichprobenumfang, Art des eingesetzten Ausgleichsverfahrens, etc. einzufordern.

Das Statistikgesetz sollte zumindest vorsehen, daß dem qualifizierten Benutzer eine solche detaillierte Dokumentation nicht verwehrt werden darf. 


\section{Schaffung der inhaltlichen Voraussetzungen zur Entlastung der Respondenten}

Der politische Auftrag, ein neues Statistikgesetz vorzubereiten, kommt nicht zuletzt aus diesem Wunsch heraus. Ich möchte zwei Unteraspekte herrausheben, die unbedingt im Statistikgesetz ihren Niederschlag finden sollten.

Der Zugang zu Registern und zu administrativen Daten ist eine notwendige, aber keine hinreichende Bedingung zur Entlastung der Respondenten: Der Zugang zu Verwaltungsdaten wird nur dann zu einer Entlastung der Respondenten und zu höherer Effizienz führen, wenn eine Verpflichtung besteht, die amtliche Statistik über Natur, Umfang, Definitionen der verfügbaren Informationen in Kenntnis zu setzen, und wenn die amtliche Statistik zwingend in alle Veränderungen in der "Informationslandschaft" eingebunden wird. Eine Mindestforderung wäre die Informationspflicht an die amtliche Statistik. Sekundärstatistik braucht Kontinuität. Das Fehlen eines Merkmals kann weite Bereiche administrativer Daten für die Statistik unbrauchbar machen. Der zweite Aspekt betrifft die unverwechselbare Erkennbarkeit der amtlichen Statistik. Die unmittelbare Erkennbarkeit amtlicher Statistik, für die Meldepflicht besteht, würde wesentlich helfen, die "Response burden" zu verkleinern. 


\section{Anhänge}

\section{ANHANG 1: Normen zur Anwendung statistischer Verfahren}

\section{Declaration of Helsinki}

I. Basic Principles

1. Biomedical research involving human subjects must conform to generally accepted scientific principles and should be based on adequately performed laboratory and animal experimentation and on a thorough knowledge of the scientific literature.

\section{Verordnung (EC) Nr. 322/97 des Rates vom 17. Februar 1997 über die Gemein- schaftsstatistiken}

“Zuverlässigkeit" bedeutet, daß die Gemeinschaftsstatistiken die Gegebenheiten, die sie darstellen sollen, so genau wie möglich widerspiegeln. Dies verlangt die Heranziehung wissenschaftlicher Kriterien bei der Wahl der Quellen, Methoden und Verfahren. Die Zuverlässigkeit der Daten ist auch durch Angaben über den Erhebungsumfang, die Methodik, die Verfahren und die Quellen zu verbessern.

\section{ANHANG 2: Anforderungen an den "Prüfer" im Österreichischen Arzneimittelgesetz}

\section{Prüfer}

$\S 35$. (1) Prüfer darf nur ein Arzt sein, der zur selbständigen Ausübung des ärztlichen Berufes in Österreich berechtigt ist, und ... 3. ... über Kenntnisse auf den einschlägigen Gebieten der nichtklinischen Medizin, insbesondere auch über Biometrie, verfügt.

(2) ... Falls der Prüfer auf einem der Teilgebiete gemäß Abs.1 Z 3 nicht die entsprechenden Kenntnisse und Erfahrungen besitzt, sind von ihm einschlägige Fachkräfte mitverantwortlich beizuziehen.

\section{ANHANG 3: Transparenz der Daten im Österreichischen Arzneimit- telgesetz}

$\S 36$. Der Prüfer hat ...

8. die Daten korrekt zu erheben, festzuhalten und zu berichten, den Umgang mit der verschlüsselten Zuordnung zu Behandlungsgruppen und die Dokumentation darüber mit äußerster Sorgfalt handzuhaben und sicherzustellen, daß der Behandlungsschlüssel ausschließlich unter den im Prüfplan angegebenen Umständen gebrochen wird, ...

10. alle Daten im Zusammenhang mit der klinischen Prüfung zum Zweck eines Audits oder einer Inspektion zugänglich zu machen, und 
11. die Erstellung des Abschlußberichtes der klinischen Prüfung zu gewährleisten und diesen zu unterzeichnen.

\section{ANHANG 4: Rolle der Biometrie im Österreichischen Arzneimittelge- setz}

Planung, Durchführung und Auswertung einer klinischen Prüfung

§37. (1) Jeder klinischen Prüfung ist ein Prüfplan zu Grunde zu legen, der Auswertbarkeit und Reproduzierbarkeit der Ergebnisse der klinischen Prüfung gewährleistet und alle für die Fragestellung relevanten Kriterien zu enthalten hat.

(2) Die Möglichkeit der biometrischen Beurteilung muß vor Beginn und während des gesamten Ablaufes einer klinischen Prüfung gegeben sein.

(3) Der Prüfplan hat insbesondere folgende Punkte zu beinhalten:

1. Definition des zu untersuchenden Zielkriteriums oder der zu untersuchenden Zielkriterien,

2. Beschreibung des Prüfdesigns und der statistisch erforderlichen Fallzahl oder Fallzahlen,

3. Dauer der klinischen Prüfung und Abbruchkriterien,

4. Ein- und Ausschlußkriterien und

5. Kriterien für Begleittherapien.

(4) Die Art der statistischen Analyse, die angewendet werden soll, muß im Prüfplan ausgeführt sein. Spätere Abweichungen von dieser Planung müssen beschrieben und im Endbericht gerechtfertigt werden.

\section{ANHANG 5: Transparenz der Methoden im Österreichischen Arznei- mittelgesetz}

§37. (5) Die Planung der statistischen Analyse und ihre Ausführung muß durch einen entsprechend qualifizierten Biometriker oder Statistiker durchgeführt oder bestätigt werden. Die Möglichkeit und die Umstände einer Zwischenauswertung müssen ebenfalls im Prüfplan ausgeführt sein.

(6) Die statistische Analyse muß Angaben über fehlende, nicht verwertete und fehlerhafte Daten enthalten.

(7) Jede Publikation einer klinischen Prüfung hat Angaben darüber zu enthalten, wer Sponsor dieser klinischen Prüfung war. 


\section{ANHANG 6: Themen der ICH-Guideline "Scientific Principles for Cli- nical Trials"}

As a starting point, this guideline utilised the CPMP Note for Guidance entitled "Biostatistical Methodology in Clinical Trials in Applications for Marketing Authorisations for Medicinal Products" (December, 1994). It was also influenced by "Guidelines on the Statistical Analysis of Clinical Studies" (March, 1992) from the Japanese Ministry of Health and Welfare and the U.S. Food and Drug Administration document entitled "Guideline for the Format and Content of the Clinical and Statistical Sections of a New Drug Application" (July, 1988). Some topics related to statistical principles and methodology are also embedded within other ICH guidelines, particularly those listed below. The specific guideline that contains related text will be identified in various sections of this document. E1: The Extent of Population Exposure to Assess Clinical Safety,

E2a: Clinical Safety Data Management: Definitions and Standards for Expedited Reporting,

E2b: Clinical Safety Data Management: Data Elements for Transmission of Individual Case Safety Reports,

E2c: Clinical Safety Data Management: Periodic Safety Update Reports for Marketed Drugs,

E3: Structure and Content of Clinical Study Reports,

E4: Dose Response Information to Support Drug Registration,

E5: Ethnic Factors in the Acceptability of Foreign Clinical Data,

E6: Good Clinical Practice: Consolidated Guideline,

E7: Studies in Support of Special Populations: Geriatrics,

E8: General Considerations for Clinical Trials,

E10: Choice of Control Group in Clinical Trials,

M1: Standardisation of Medical Terminology for Regulatory Purposes,

M3: Non-Clinical Safety Studies for the Conduct of Human Clinical Trials for Pharmaceuticals.

\section{ANHANG 7: Inhaltsverzeichnis der ICH-Guideline "Scientific Pricip- les for Clinical Trials"}

I. INTRODUCTION

1.1 Background and Purpose

1.2 Scope and Direction

II CONSIDERATIONS FOR OVERALL CLINICAL DEVELOPMENT

2.1 Study Context

2.1.1 Development Plan

2.1.2 Confirmatory Trial

2.1.3 Exploratory Trial

2.2 Study Scope 


\subsubsection{Population}

2.2.2 Primary and Secondary Variables

2.3 Design Techniques to Avoid Bias

2.3.1 Blinding

2.3.2 Randomisation

III. STUDY DESIGN CONSIDERATIONS

3.1 Study Configuration

3.1.1 Parallel Group Design

3.1.2 Cross-Over Design

3.1.3 Factorial Design

3.2 Multicentre Trials

3.3 Type of Comparison

3.3.1 Trials to Show Superiority

3.3.2 Trials to Show Equivalence or Non-Inferiority

3.3.3 Dose-response Designs

3.4 Group Sequential Designs

3.5 Sample Size

3.6 Data Capture and Processing

\section{STUDY CONDUCT}

4.1 Trial Monitoring

4.2 Changes in Inclusion and Exclusion Criteria

4.3 Accrual Rates

4.4 Sample Size Adjustment

4.5 Internal Analysis and Early Stopping

4.6 Role of Independent Data Monitoring Committee

\section{DATA ANALYSIS}

5.1 Prespecified Analysis Plan

5.2 Analysis Sets

5.2.1 All Randomised Subjects

5.2.2 Per Protocol Subjects

5.2.3 Roles of the All Randomised Subjects Analysis and the Per Protocol Analysis 5.3 Missing Data and Outliers

5.4 Data Transformation/Modification

5.5 Estimation, Confidence Intervals and Hypothesis Testing

5.6 Adjustment of Type I Error and Confidence Levels

5.7 Subgroups, Interactions and Covariates

5.8 Integrity of Data and Computer Software

\section{EVALUATION OF SAFETY AND TOLERABILITY}

6.1 Scope of Evaluation 
6.2 Choice of Variables and Data Collection

6.3 Set of Subjects to be Evaluated and Presentation of Data

6.4 Statistical Evaluation

6.5 Single Study vs. Integrated Summary

\section{REPORTING}

7.1 Evaluation and Reporting

7.2 Summarising the Clinical Databasis

7.2.1 Efficacy Data

7.2.2 Safety Data

\section{ANHANG 8: ICH-Guideline: 4.4 Sample Size Adjustment}

In long term trials there will usually be an opportunity to check the assumptions which underlay the original design and sample size calculations. This may be particularly important if the trial specifications have been made on preliminary and/or uncertain information. An interim check conducted on the blinded data may reveal that overall response variances, event rates or survival experience are not as anticipated. A revised sample size may then be calculated using suitably modified assumptions, and should be justified and documented in a protocol amendment and in the final report. The steps taken to preserve blindness and the consequences, if any, for the type I error and the width of confidence intervals should be explained. The potential need for re-estimation of the sample size should be envisaged in the protocol whenever possible (see Section 3.5). 